\title{
$A b$ initio simulations and measurements of the free-free opacity in aluminum
}

\author{
P. Hollebon, O. Ciricosta, M. P. Desjarlais, C. Cacho, C. Spindloe, \\ E. Springate, I. C. E. Turcu, J. S. Wark and S. M. Vinko
}

\section{Published version information}

Citation: P Hollebon et al. "Ab initio simulations and measurements of the free-free opacity in aluminum." Physical Review E, vol. 100, no. 4 (2019): 043207.

DOI: $10.1103 /$ PhysRevE.100.043207

This version is made available in accordance with publisher policies. Please cite only the published version using the reference above. This is the citation assigned by the publisher at the time of issuing the APV. Please check the publisher's website for any updates. 


\title{
$A b$ initio simulations and measurements of the free-free opacity in aluminum
}

\author{
P. Hollebon,,${ }^{1, *}$ O. Ciricosta, ${ }^{1}$ M. P. Desjarlais,${ }^{2}$ C. Cacho,${ }^{3}$ C. Spindloe,${ }^{3}$ E. Springate,${ }^{3}$ I. C. E. Turcu,${ }^{3}$ \\ J. S. Wark, ${ }^{1}$ and S. M. Vinko ${ }^{1, \dagger}$ \\ ${ }^{1}$ Department of Physics, Clarendon Laboratory, University of Oxford, Parks Road, Oxford OX1 3PU, United Kingdom \\ ${ }^{2}$ Pulsed Power Sciences Center, Sandia National Laboratories, Albuquerque, New Mexico 87185, USA \\ ${ }^{3}$ Central Laser Facility, STFC Rutherford Appleton Laboratory, Didcot OX11 OQX, United Kingdom
}

(Received 23 March 2018; revised manuscript received 21 March 2019; published 23 October 2019; corrected 31 October 2019)

\begin{abstract}
The free-free opacity in dense systems is a property that both tests our fundamental understanding of correlated many-body systems, and is needed to understand the radiative properties of high energy-density plasmas. Despite its importance, predictive calculations of the free-free opacity remain challenging even in the condensed matter phase for simple metals. Here we show how the free-free opacity can be modelled at finite-temperatures via time-dependent density functional theory, and illustrate the importance of including local field corrections, core polarization, and self-energy corrections. Our calculations for ground-state Al are shown to agree well with experimental opacity measurements performed on the Artemis laser facility across a wide range of extreme ultraviolet wavelengths. We extend our calculations across the melt to the warm-dense matter regime, finding good agreement with advanced plasma models based on inverse bremsstrahlung at temperatures above $10 \mathrm{eV}$.
\end{abstract}

DOI: 10.1103/PhysRevE.100.043207

\section{INTRODUCTION}

The mechanisms by which free electrons in a plasma absorb and emit radiation are of key importance to a range of applications, from investigations of laser-plasma interactions to astrophysics and inertial confinement fusion research. The free-free opacity in classical plasmas is generally described using the inverse bremsstrahlung (IB) formalism, initially treated classically by Kramers [1], and later modified to include a range of additional corrections to the absorption cross section including quantum effects [2], multiphoton contributions [3], relativistic corrections [4], electron degeneracy [5], and collective phenomena [6-8]. These models contain Coulomb logarithm terms to describe electron interactions and are typically limited in applicability to plasmas where small-angle collisions dominate energy transfer in the electron subsystem, i.e., plasmas at high temperatures and low densities.

Dense plasmas, in turn, have proven far more challenging both to model and to investigate experimentally [9-14]. Perhaps surprisingly, similar difficulties are encountered in condensed matter systems such as ground-state and liquid metals. Here the absorption process is generally treated using linear response theory via calculations of the dielectric function or the complex conductivity $[15,16]$. Because the dielectric function provides a full description of the system's response, opacity investigations can be used to validate $a b$ initio models more generally, and provide stringent constraints on the approximations deployed to study correlated many-body systems [17-21]. In particular, simple metals irradiated at photon energies below their bound-state ionization edges are

\footnotetext{
*patrick.hollebon@physics.ox.ac.uk; phollebon@lanl.gov

†sam.vinko@physics.ox.ac.uk
}

ideal candidates to investigate the fundamental mechanisms of free-free opacity, and therefore of the dielectric response. Such systems are essentially ground-state plasmas, both simple to manipulate and investigate experimentally, and are present in well-defined conditions of temperature, density, and ionization.

Density functional theory (DFT) calculations are invaluable for providing a single computational framework in which to model quantum mechanical systems across a wide variety of temperatures and densities. More explicitly, multi-atom Kohn-Sham DFT calculations coupled with quantum molecular dynamics (MD) have provided accurate simulations of equilibrium properties within both periodic and disordered systems through the use of large supercells. Excited states and response properties are also accessible via time-dependent DFT and perturbation theory. DFT is thus well placed as a natural starting point when looking for a single framework, and associated set of approximations, in which to consistently model multiple physical properties across a range of experimentally accessible conditions.

Despite this, both the theoretical and the experimental free-free absorption cross sections in $\mathrm{Al}$, a simple metal, remain poorly understood. While calculations based on the random phase approximation (RPA) are seen to be in good agreement with bound-state opacity measurements, the RPA performs poorly in the free-free regime for ground-state $\mathrm{Al}$ [18]. Furthermore, there is a significant discrepancy in the experimental free-free opacity in the XUV photon energy range between the $\mathrm{Al}$ plasma frequency at $15 \mathrm{eV}$ and the $\mathrm{Al}$ L-edge at $73 \mathrm{eV}$. The widely used Centre for X-Ray Optics online database (CXRO) [22] uses the experimental data of Gullikson et al. [23] for this energy range; results which disagree by as much as a factor of 2 with measurements by Henke et al. [24] and Keenan et al. [25]. Given that thin Al foils are commonly used as filters in the XUV, such discrepancies can 


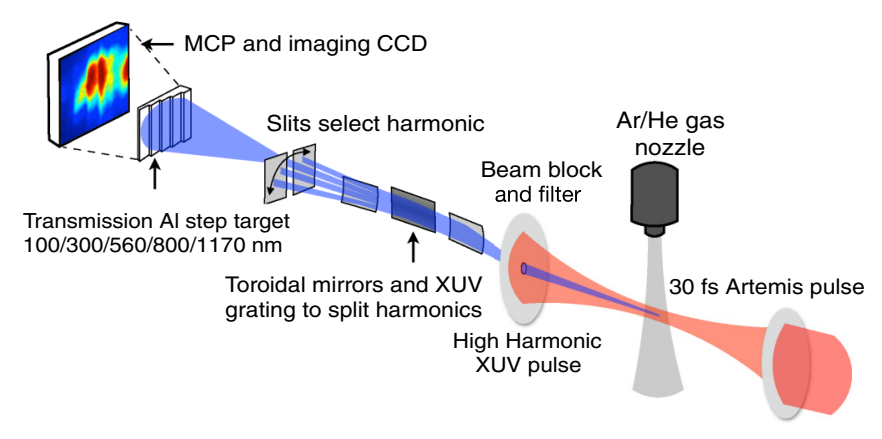

FIG. 1. Experimental setup for the opacity measurement on the Artemis facility.

have a large effect on the calculated spectral brightnesses of XUV sources created via high harmonic generation (HHG) or other techniques.

Here we present experimental measurements and ab initio results for the ground-state $\mathrm{Al}$ free-free opacity. Our calculations are based on a first principles simulations of the electronic response tensor using DFT. By contrast, in the literature macroscopic, optical properties of warm-dense matter systems are routinely computed inserting the Kohn-Sham states into the long-wavelength limit of the Kubo-Greenwood formula alone. Our calculations move beyond this approximation by incorporating both local field corrections (LFC) and a finite-temperature, many-body theory treatment of the singleparticle self-energy: neither of which have been typically accounted for in standard, DFT Kubo-Greenwood calculations for the dynamic conductivity warm-dense matter.

Our calculations are compared to new experimental results obtained by measuring the attenuation of an HHG source through stepped targets. In this manner, we may clearly distinguish absorption by the target from that due to impurities or oxide layers that exist on the surface [26]. Excellent agreement is found when we account for LFC and many-body corrections to the energy of the Al L-edge; the second of these being underestimated in the DFT-only calculation.

The consequences of LFC and finite-temperature quasiparticle corrections for the free-free opacity are examined for warm dense matter conditions of solid density $\mathrm{Al}$ at temperatures of $1,5,10$, and $15 \mathrm{eV}$. We find our calculations approach advanced IB-based plasma models at higher temperatures. The inclusion of finite-temperature many-body corrections has necessitated the modification of the standard ABINIT DFT code, within which we have implemented our own version of a finite-temperature $\mathrm{G}_{0} \mathrm{~W}_{0}$ quasiparticle code.

\section{EXPERIMENTAL MEASUREMENTS}

The experiment was conducted at the HHG Artemis facility [27] of the Rutherford Appleton Laboratory, UK, capable of delivering XUV pulses in the XUV spectral range between $15-60 \mathrm{eV}$. The setup is shown schematically in Fig. 1. Here, a 1-kHz Ti:sapphire CPA laser system provided short (30-fs FWHM) infrared pulses at a wavelength of $780 \mathrm{~nm}$. These pulses were focused onto an $\mathrm{Ar}$ or Ne gas jet within a differentially pumped gas cell at intensities around $10^{14} \mathrm{~W} / \mathrm{cm}^{2}$ to produce high harmonics over a relatively broad spectrum, with an energy efficiency of order $10^{-6}$. Individual harmonics were picked out from the HHG spectrum via a time-preserving XUV monochromator with a resolving power $\lambda / \Delta \lambda \approx 100$ and peak transmissivity of 20\% [27], and were steered onto an $\mathrm{Al}$ target using a gold mirror. The transmitted beam through the target hits a microchannel plate (MCP) detector and was imaged with a standard optical system and camera. The background signal and spatial profile of the HHG pulses was determined by imaging the beam in the absence of the sample. The linearity of the optical system was verified during the experiment across the entire dynamic range of transmitted signals and for all exposure time durations. Typical exposure times ranged from 1-5 seconds, so the data integrates over several thousand shots for each experimental opacity data point.

The target sample is a free-standing five-step foil with the different thicknesses of $\mathrm{Al}$ deposited in a single process to ensure there are no layers of spurious materials between the individual steps. The target size is about 3 by $4 \mathrm{~mm}$, and is fully illuminated by the diverging HHG beam from the source (Fig. 1). Thicknesses of the various steps vary slightly with position but are on average 100, 315, 560, 810 , and $1165 \mathrm{~nm}$, measured via profilometry to an accuracy better than $2 \mathrm{~nm}$. Using a single Al sample with a range of thicknesses is crucial because surface contaminants and oxide layers can have a large effect on the absorption, given the vastly different attenuation lengths in the XUV regime. By looking at the differential absorption across the steps we can overcome the well-known difficulties related to surface oxide layers and other contaminants commonly present on thin foils. The absorption of XUV light in the low-intensity limit follows the Beer-Lambert law:

$$
T(x, y)=\exp (-\kappa(\omega) d(x, y)-\alpha(\omega)) .
$$

Here $T$ is the experimentally measured transmission through a specific point of the target denominated by $(x, y)$. The thickness of the absorbing $\mathrm{Al}$ target at that point is $d(x, y), \kappa(\omega)$ is the frequency-dependent absorption coefficient of $\mathrm{Al}$, and $\alpha(\omega)$ is the frequency-dependent absorption term (including thickness) representing any additional contributions to the absorption, here predominantly arising from the aluminum oxide layer. We implicitly assume that there is no variation in oxide thickness across the target.

By taking the negative logarithm of the transmission we can write a linear equation for the absorption coefficients as a function of the variable $d$, the thicknesses of the Al step target:

$$
\kappa(\omega) d+\alpha(\omega)=-\log (T) .
$$

We show the measured attenuation through the sample in Fig. 2 for the 15th laser harmonic. The steps in the sample can easily be observed in the three-dimensional (3D) plot of the transmission. Because the sample thickness is accurately known before the experiment, we can extract experimental values for both $\kappa(\omega)$ and $\alpha(\omega)$ by fitting the data using the linear function of sample thickness given in Eq. (2). We show this procedure in Fig. 3, again for the 15th harmonic. From this analysis we find an absorption coefficient to be $\kappa(\omega)=5.1 \times 10^{6} \mathrm{~m}^{-1}$, with an uncertainty of $0.9 \times 10^{6} \mathrm{~m}^{-1}$, where we quote a $95 \%$ confidence interval, i.e., $\pm 2 \sigma$. The 

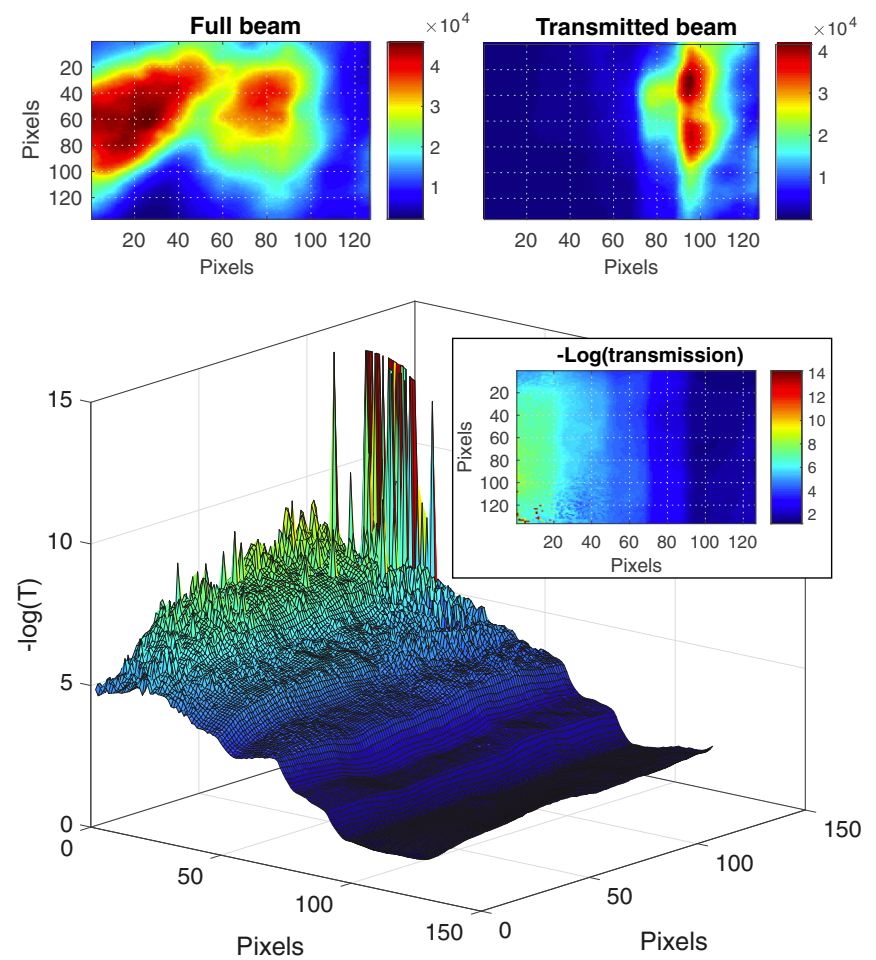

FIG. 2. Measurement of the transmission through the Al step target for the 15th laser harmonic around $24 \mathrm{eV}$. The top panels show the spatial intensity distribution of the HHG beam without a target (full beam), and the transmitted intensity through the step target. The natural $\log$ of the ratio of the two yields the total absorption [see Eq. (2)], which when plotted in 3D to illustrate that the steps of the target are clearly visible in the absorption measurement.

same procedure was repeated for harmonics 11-37, yielding an opacity dataset across the XUV range of the spectrum that we will discuss in what follows. Importantly, to obtain the experimental absorption coefficient using our approach it is only necessary to know the difference in thicknesses of the various steps, while their absolute values are inconsequential.

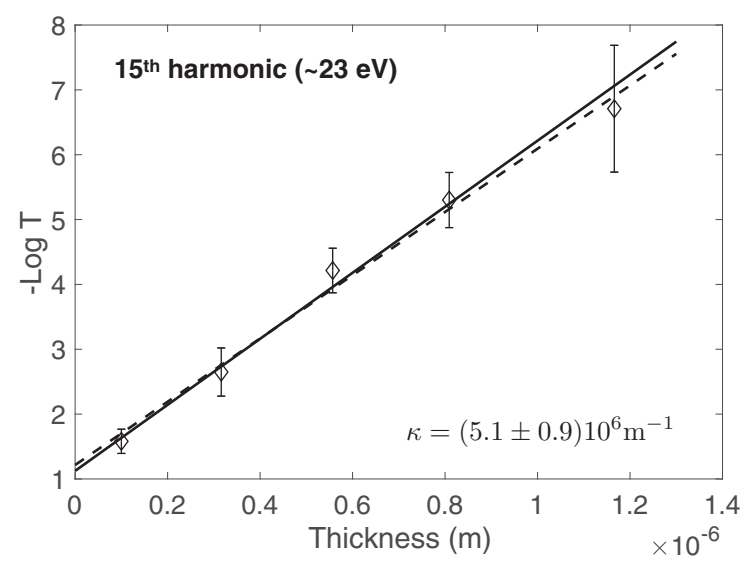

FIG. 3. Observed transmission of the 15th harmonic through the $\mathrm{Al}$ step target sample. Following Eq. (2) we perform a linear fit to the five data points to extract $\kappa(\omega)$ (the slope), $\alpha(\omega)$ (the $y$ intercept), and related errors. Fitted lines corresponding to the largest and smallest slopes are shown.
By extension, we can actually use our measured offset coefficient $\alpha(\omega)$ to determine the oxide thickness if we assume the dominant contribution to $\alpha(\omega)$ is a layer of $\mathrm{Al}_{2} \mathrm{O}_{3}$ of known opacity, such as that taken from the CXRO database [22]. Following this approach we find the thickness of the total oxide layer to be $(15 \pm 6) \mathrm{nm}$.

\section{DFT SIMULATIONS}

To determine the long wavelength absorption opacity we first consider the macroscopic dielectric function of the plasma $\epsilon_{M}$, from which the absorption coefficient follows via $\kappa(\omega)=\frac{2 \omega}{c} \operatorname{Im} \sqrt{\epsilon_{M}(\omega)}$. Our calculations begin with multicentred DFT simulations, solving the finite-temperature KohnSham equations

$$
\left[\frac{-\hbar^{2}}{2 m_{e}} \nabla^{2}+v_{\mathrm{H}}+v_{\mathrm{xc}}\right] \psi_{n}^{\mathrm{KS}}=\epsilon_{n}^{\mathrm{KS}} \psi_{n}^{\mathrm{KS}} .
$$

Where $v_{\mathrm{H}}$ and $v_{\mathrm{xc}}$ are the Hartree and exchange-correlation potentials. Electron-ion interactions are modelled using projector augmented wave (PAW) [28,29] pseudopotentials with the $1 s$ electrons frozen in the Al core. Up to 1500 bands are calculated in 32 atom unit cells with a $2 \times 2 \times 2 \mathrm{k}$-point grid Brillouin zone sampling and a planewave cutoff of $400 \mathrm{eV}$. An ensemble of ion positions is obtained by evolving the system in time for $3 \mathrm{ps}$ in the Born-Oppenheimer approximation while coupled to a Nosé-Hoover thermostat. We compare the results of these MD simulations to those average-atom and multicentred simulations for the equation of state of solid density $\mathrm{Al}$ (Fig. 4) as an additional verification.

At this point, the Kubo-Greenwood forumula is often applied to calculating the real part of the dynamic conductivity:

$$
\begin{aligned}
\sigma(\omega)= & \frac{2 \pi}{3 \omega V} \sum_{i, j=1} \sum_{\alpha=1}^{3}\left[F\left(\epsilon_{i, \mathbf{k}}^{\mathrm{KS}}\right)-F\left(\epsilon_{j, \mathbf{k}}^{\mathrm{KS}}\right)\right] \\
& \times\left|\left\langle\psi_{j, \mathbf{k}}^{\mathrm{KS}}\left|\nabla_{\alpha}\right| \psi_{i, \mathbf{k}}^{\mathrm{KS}}\right\rangle\right|^{2} \delta\left(\epsilon_{j, \mathbf{k}}^{\mathrm{KS}}-\epsilon_{i, \mathbf{k}}^{\mathrm{KS}}-\omega\right) .
\end{aligned}
$$

The imaginary component may then be obtained via a Kramer-Kronig transform, and the dielectric function

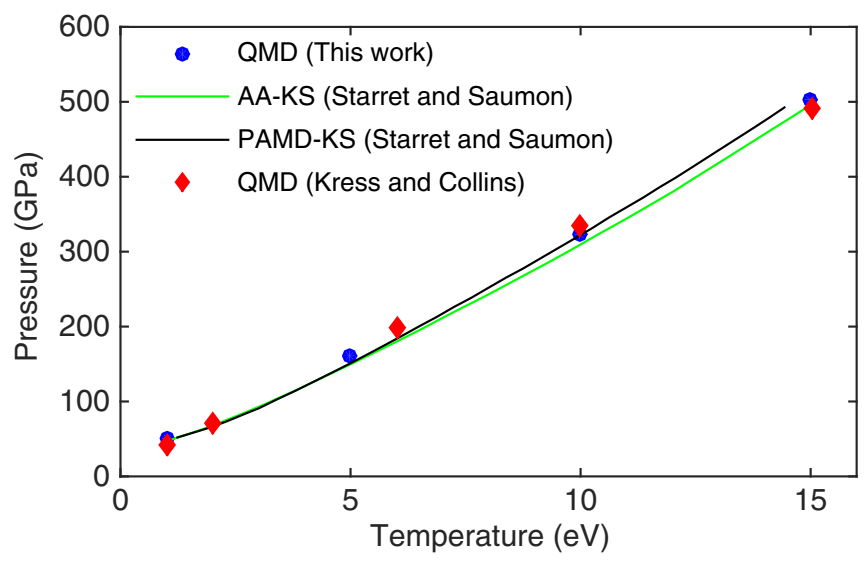

FIG. 4. Comparison of the equation of state for solid density, warm dense $\mathrm{Al}$ as predicted following our MD simulations, confirming consistency with previously published average-atom and multicentred Kohn-Sham calculations [30]. 
determined through the relation

$$
\epsilon(q \rightarrow 0, \omega)=1+\frac{i \sigma(\omega)}{\omega} .
$$

This procedure, however, does not account for two important contributions to the macroscopic dielectric functions that appear in a rigorous approach based on a time-dependent DFT response theory, namely the role of LFC and changes in the exchange-correlation potential.

Starting with the former, we can elucidate the role of LFC if we first note that in our calculations we have access to the full dielectric tensor for a given snapshot during the MD simulation. In a periodic system we can write this in the form $\epsilon_{G G^{\prime}}(q, \omega)$, where $G$ and $G^{\prime}$ are reciprocal lattice vectors, and $q$ lies within the first Brillouin zone. $\epsilon_{M}$ is then defined via

$$
\epsilon_{\mathrm{mac}}(\omega)=\frac{1}{\epsilon_{00}^{-1}(q \rightarrow 0, \omega)} \neq \epsilon_{00}(q \rightarrow 0, \omega) .
$$

By contrast, the Kubo-Greenwood formula even with the exact many-body eigenstates $\Psi\left(\mathbf{r}_{1}, \mathbf{r}_{2}, \mathbf{r}_{3}, \ldots\right.$, ) provides only the head of the tensor $\epsilon_{00}$.

The difference between the left and right sides of the inequality in Eq. (6) comprises the LFC contribution, and vanishes only in the limit that the dielectric tensor is a function of separation distance, or alternatively $q$, only. For a molten system, once we average over an ensemble of nuclei positions taken from an MD simulation no particular position should be special. Thus in the thermodynamic limit quantities must depend only on relative distance. However, provided that the absorption takes place on a timescale much shorter than nuclei motion, each instantaneous configuration of nuclei positions during the MD corresponds to a specific external potential, one "local" to that state in the thermodynamic ensemble, generated by a specific spatial configuration of nuclei under which the absorption takes place. Physically, ignoring LFC in this context is equivalent to applying a form of random phase approximation (RPA) to electron-nuclei scattering; thereby neglecting the instantaneous nuclei-nuclei spatial correlations as has been previously done, for example, in Ref. [18].

In addition to LFC, a complete calculation of linear response properties must include changes in the exchangecorrelation potential to account for the difference between the Kohn-Sham orbitals and the true many-body states. Timedependent DFT provides such a means to formally calculate the first order, dynamic response properties of finitetemperature systems via the exchange-correlation kernel $f_{\mathrm{xc}}$. If we define the linear density response function of the (noninteracting) Kohn-Sham system as $\chi^{0}$, then the correct, interacting density response $\chi$ can be obtained from

$$
\chi=\frac{\chi^{0}}{1-K \chi^{0}}, \quad K=V_{c}+f_{\mathrm{xc}} .
$$

Here $V_{c}$ is the Hartree-Coulomb term, and both frequency dependence and the tensor nature of the quantities is suppressed for brevity. In reality, response function calculations are limited by the availability of suitable approximations to $f_{\mathrm{xc}}$, which is, in general, temperature dependent, timeenergy dependent, and a functional of the current density. This is particularly relevant when dealing with dynamic properties of systems at finite temperatures [21,31], which require explicitly time [32-34] and temperature-dependent functionals [35-39]. Furthermore, from a practical perspective whether or not common static exchange-correlation kernels (for example, local density and generalized gradient approximations) provide any advantage over completely neglecting this term is somewhat situation dependent.

Rather than rely on approximate DFT exchange-correlation kernels in the next section we will instead use many-body theory to correct the Kohn-Sham states and raise them to the a physical level of quasiparticles. To do this, however, and for comparative purposes with the final result, we first calculate the response properties neglecting the exchange-correlation kernel $f_{\mathrm{xc}} \approx 0$.

$$
\begin{aligned}
\chi & =\frac{\chi^{0}}{1-V_{c} \chi^{0}}, \quad K \approx V_{c} \\
\epsilon^{-1} & =1+V_{c} \chi \Rightarrow \epsilon=1-V_{c} \chi^{0} .
\end{aligned}
$$

By keeping only the Hartree-Coulomb contribution to the kernel $K$, Eq. (8) is in effect an RPA approximation with respect to electron-electron correlations during the dynamic response. Note that these electron-electron correlations are still, albeit approximately, accounted for during the initial DFT calculation for the Kohn-Sham system. Aside from the aforementioned ambiguous nature of the benefits of available exchange-correlation functions, the approximation of $f_{\mathrm{xc}} \approx 0$ at this stage is taken also with the knowledge that much of the relevant exchange-correlation physics will be restored when the resulting dielectric tensor is used to perform quasiparticle calculations in the next section.

We used the ABINIT code $[40,41]$ to calculate the KohnSham wave functions via Eq. (3) and to compute the dielectric function $[42,43]$ using the above response theory formalism. Special care was taken in dealing with the long wavelength limit of intraband contributions to the dielectric function for the purpose of satisfying sum rules. To this end, we explicitly calculated the limit of $\epsilon(q \rightarrow 0, \omega \rightarrow 0)_{00}$ by fitting the low-frequency response of the dielectric function at finite wavevector using a single plasmon-pole approximation

$$
\epsilon^{-1}(q, \omega)=1+\frac{\left(\omega_{p}^{0}\right)^{2}}{\omega\left(\omega+i \nu^{q}\right)-\left(\omega_{p}^{q}\right)^{2}},
$$

where the plasma frequency $\omega_{p}^{q}$ and broadening $\nu^{q}$ are $q$ dependent. We found the dielectric response at low frequencies to be well fitted by this functional form for all conditions studied. The $q \rightarrow 0$ plasma frequency, including both inter and intraband contributions, was then obtained by fitting for $\omega_{p}^{q}$ and extrapolating via our calculated Bohm-Gross relation for $\omega_{p}^{q}$ to $q=0$.

\section{FINITE-TEMPERATURE QUASIPARTICLE CORRECTIONS}

LFC effects may physically be expected to be important for systems with strong electron-nuclei and nuclei-nuclei correlations. As mentioned, electron-electron correlations can, in principle, be taken into account with suitable exchangecorrelation kernels, the development and testing of which continues. However, within condensed matter an established 
and accurate alternative has been to use DFT calculations as the starting point for many-body theory calculations. In particular, the GW approximation for the single-particle selfenergy has been widely applied to fixing the so-called bandgap problem of DFT in which the Kohn-Sham eigenvalues regularly underestimate energy gaps in the density of states of extended systems [44]. In the context of our calculations here, this applies most immediately to the position of the absorption L-edge in the opacity of $\mathrm{Al}$, a transition featuring bound states strongly coupling to the nuclei and thus, perhaps more susceptible to LFC than contributions from valence-valence transitions in our free-electron-like metal.

The simplest many-body correction we can perform is a "one-shot" cycle of Hedin's equations [45,46] referred to as the $\mathrm{G}_{0} \mathrm{~W}_{0}$ correction to the Kohn-Sham states. Such calculations are relatively standard for ground-state systems, however, here we apply them at finite temperatures [47] using our custom code to study warm dense matter systems via many-body theory corrections to initial DFT calculations. GW and other approximations for the self-energy have, however, previously been applied to plasma physics independently of DFT [48-55].

The primary computational hurdle of the many-body approach is the calculation of the correlation energy $\Sigma^{C}(\omega)$, with which we can define the self-energy $\Sigma=\Sigma^{X}+\Sigma^{C}$, where $\Sigma^{X}$ is the finite-temperature exchange term. This then features in the effective Schrödinger equation for quasiparticle states $\psi^{\mathrm{QP}}$ :

$$
\left[\frac{-\hbar^{2}}{2 m_{e}} \nabla^{2}+v_{\mathrm{H}}\right] \psi_{n}^{\mathrm{QP}}+\int d \mathbf{r}^{\prime} \Sigma\left(\mathbf{r}, \mathbf{r}^{\prime}, \epsilon_{n}\right) \psi_{n}^{\mathrm{QP}}\left(\mathbf{r}^{\prime}\right)=\epsilon_{n} \psi_{n}^{\mathrm{QP}} .
$$

For our single-shot $\mathrm{G}_{0} \mathrm{~W}_{0}$ calculations we approximate the self-energy $\Sigma$ as a small perturbation to the Kohn-Sham exchange correlation potential $v_{\mathrm{XC}}$ of Eq. (3). The effect of which on the density of states is described through the diagonal elements of the spectral function $A_{n n}(\omega)$ in the KohnSham basis $\psi_{n}^{\mathrm{KS}}$ :

$$
A_{n n}(\omega)=\frac{\operatorname{Im} \Sigma_{n n}(\omega)}{\left[\hat{\epsilon}_{n}+\operatorname{Re} \Sigma_{n n}(\omega)-\omega\right]^{2}+\left[\operatorname{Im} \Sigma_{n n}(\omega)\right]^{2}} .
$$

Here $\hat{\epsilon}_{n}$ are the Kohn-Sham eigenvalues minus the contributions to from the exchange-correlation potential.

In our calculations we make use of the Matsubara formalism to first express $\Sigma^{C}(z)$ for points $z=i v_{n^{\prime}}=\left(2 n^{\prime}+1\right) \pi i$ along the imaginary axis in terms of the dielectric function at frequencies $i \omega_{n}=2 n \pi i$ :

$\Sigma_{n n \mathbf{k}}^{C}\left(i v_{n^{\prime}}\right)=\frac{i T}{2 \pi} \sum_{G G^{\prime}, m, i \omega_{n}} \frac{\left[M_{G}^{m n}(\mathbf{k})\right]^{*} M_{G^{\prime}}^{m n}(\mathbf{k}) W_{G G^{\prime}}^{C}\left(\omega_{n}\right)}{i v_{n^{\prime}}+i \omega_{n}-\epsilon_{m}^{\mathrm{KS}}(\mathbf{k})}$,

where the Kohn-Sham matrix elements are $M_{G}^{m n}=\left\langle\psi_{m}^{\mathrm{KS}}(\mathbf{k})\right|$ $e^{i G r}\left|\psi_{n}^{\mathrm{KS}}(\mathbf{k})\right\rangle$, the screened Coulomb interaction is given by $W_{G G^{\prime}}^{C}(\omega)=\left[\epsilon_{G G^{\prime}}^{-1}(\omega)-1\right] V_{G G^{\prime}}^{C}$, and where we set the chemical potential $\mu=0$ for convenience.

The retarded self-energy $\Sigma\left(\omega+i 0^{+}\right)$is then obtained by analytical continuation from the upper-half plane to the real axis. In our calculation we achieve this numerically by fitting to a Padé approximant. In condensed matter systems

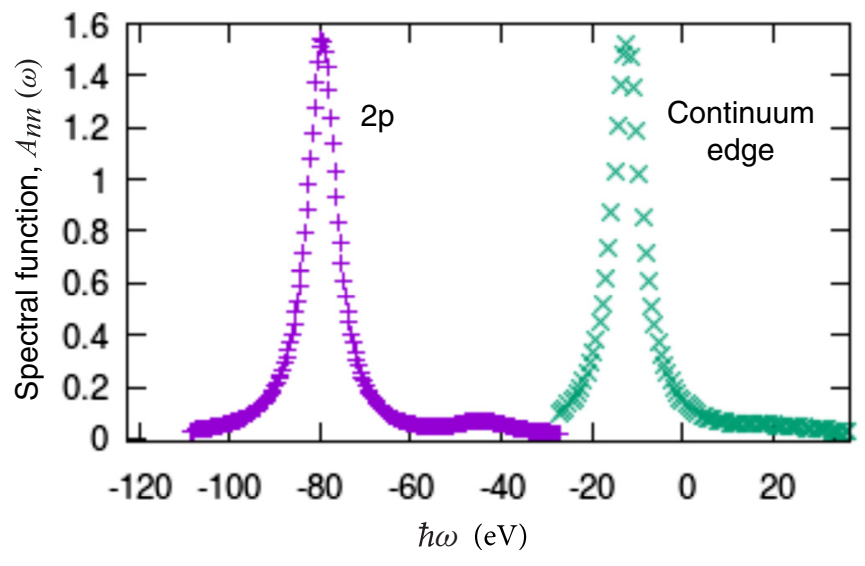

FIG. 5. Spectral function for the $2 p$ bound state and the lowest lying continuum state for $T=15 \mathrm{eV}$. Restriction to single shot $\mathrm{G}_{0} \mathrm{~W}_{0}$ combined with a finite-order Padé approximant for the analytic continuation results in broad quasiparticle peaks lacking clear satellite features.

this method of numerical analytic continuation is normally considered to be a less accurate, but quicker, alternative to the contour deformation method [56]. We find it to be sufficient for its primary purpose here of correcting the position of the L-edge.

In Fig. 5 we plot example spectral function matrix elements $A_{n n}(\omega)$ corresponding to the bound $2 p$ and lowest energy continuum state in $15 \mathrm{eV}$ Al plasma. The spectral function describes the contribution from each single particle wave function to the density of states. Restriction to single shot $\mathrm{G}_{0} \mathrm{~W}_{0}$ combined with a finite-order Padé approximant for the analytic continuation results in overly broad quasiparticle peaks lacking clear satellite features. As such, considering that our primary motivation here is to correct band gaps, we thereby choose to neglect quasiparticle lifetimes in our calculations and instead use the calculated spectral function to determine new, $\mathrm{G}_{0} \mathrm{~W}_{0}$ corrected, single particle energies by fitting each quasiparticle peak to a Lorentzian. On the same note, while $\mathrm{GW}$ is frequently used in the ground state to correct band gaps to sub-eV accuracy [44], a detailed study of finite-temperature many-body effects on the structure within the continuum, which may well require a much more accurate treatment of the spectral function shape than that afforded by our Padé approximant [55,57-59], is beyond the scope of this paper.

Having now obtained the $\mathrm{G}_{0} \mathrm{~W}_{0}$ corrected, single particle energies, we can then recalculate the dielectric function, and thus determine the opacity, following the formalism of the previous section. Our quasiparticle calculation allows for an account of exchange and correlation physics not easily modelled in DFT, such as when determining energy gaps. As such, while neglecting $f_{\mathrm{xc}}$ in the initial dielectric function calculation may be considered an approximation, when recalculating the dielectric function with our quasiparticle eigenenergies it would be incorrect to include a DFT kernels $f_{\mathrm{xc}}$.

\section{RESULTS AND DISCUSSION}

We plot our experimental results alongside previous measurements and our DFT-based many-body-theory calculations 


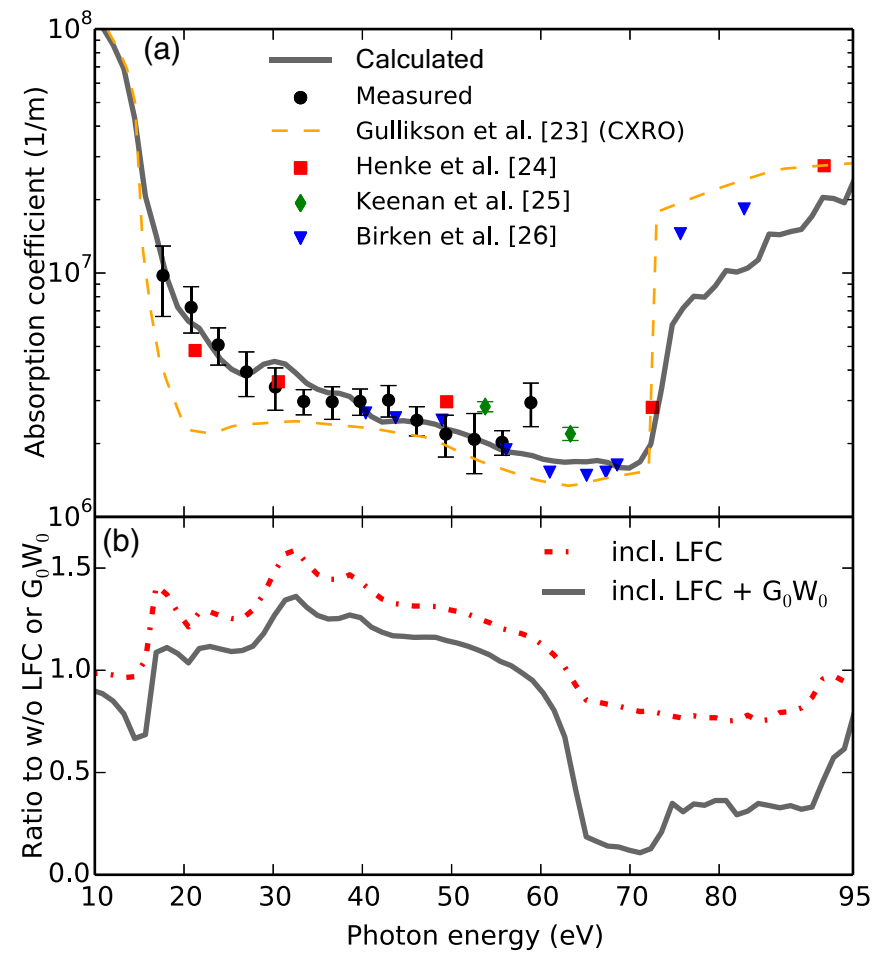

FIG. 6. (a) Calculated opacity for room temperature Al along with our own measurements and those of previous studies. (b) LFC increases absorption between the plasma frequency and the L-edge, while the primary effect of the $\mathrm{G}_{0} \mathrm{~W}_{0}$ correction is to shift the energy of the L-shell reducing the opacity.

in Fig. 6(a). We note good agreement between our measurements and those of Henke et al. [24], and Birken et al. [26], but a clear disagreement with the recently reported measurement of Kettle et al. [12], and with the data by Gullikson et al. [23] (CXRO) at photon energies below $\sim 30-40 \mathrm{eV}$. Our results also appear broadly consistent with the recently reported cold opacity measurements by Williams et al. [14] $\left(\kappa=2.5 \times 10^{6} \mathrm{~m}^{-1}\right)$, though as the latter measurement was not frequency resolved a more complete comparison is not possible.

The contribution of the LFC and of $\mathrm{G}_{0} \mathrm{~W}_{0}$ on the roomtemperature opacity calculations is shown in Fig. 6(b). The LFC overall raises the opacity between the plasma frequency and the L-edge, while the effect of the $\mathrm{G}_{0} \mathrm{~W}_{0}$ correction is to shift the L-edge to the correct L-shell binding energy, and to slightly decrease the opacity. The full calculation including LFC and $\mathrm{G}_{0} \mathrm{~W}_{0}$ agrees well with our measured free-free opacity and the experimental position of the L-edge. However, our calculations appear to underestimate the opacity immediately above the L-edge but show signs of approaching the CXRO database values at higher photon energies.

We show in Fig. 7 our calculations extended into the warm dense matter regime and plot the predicted opacity for equilibrium, solid density $\mathrm{Al}$ at $T=1,5,10$, and $15 \mathrm{eV}$. In moving from room temperature to $T=1 \mathrm{eV}$ the free-free opacity increases considerably. This is in contrast to previous averageatom [13], as well as predicting a greater increase compared to recent IB calculations [10], and being in disagreement with the recent measurement of Kettle et al. [12]. We note,

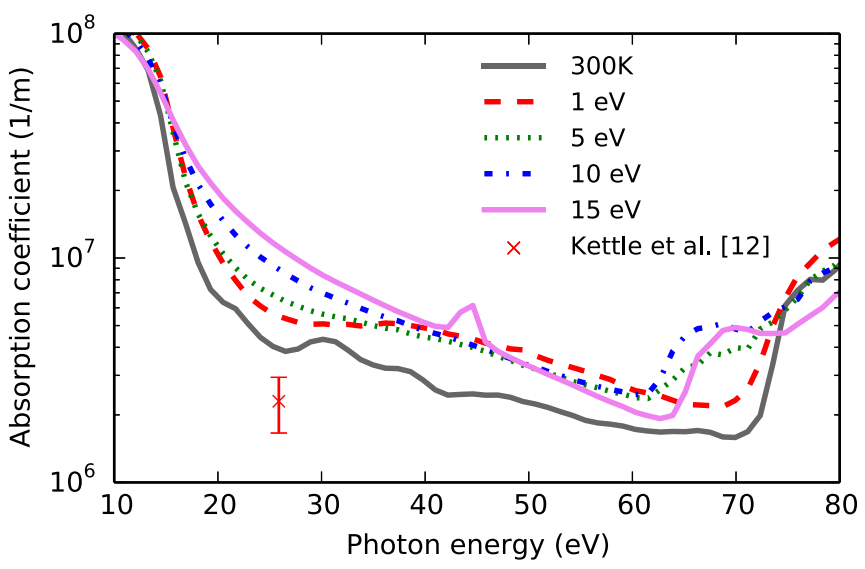

FIG. 7. Calculated opacity for equilibrated warm dense Al up to $T=15 \mathrm{eV}$ along with the single data point of Kettle et al. $\left(T_{e} \approx 1 \mathrm{eV}\right)$.

however, that the cold opacity result from the same authors is also in disagreement with several experimental datasets, as well as with our theoretical predictions.

Calculations performed using room temperature ion configurations, but with the electrons heated to $1 \mathrm{eV}$, suggest a breakdown of the regular crystalline lattice to be responsible for the sudden increase in free-free absorption at $T=1 \mathrm{eV}$. This may explain why such an increase was not predicted by the previous IB theory (in which the role of ion-ion correlations was only estimated) or average-atom calculations. A key difference between this last and our calculations is the use here of periodic supercells, well suited to representing the periodic $\mathrm{Al}$ fcc crystal structure in the cold limit, and capable of dealing with nonspherically symmetric ion distributions. The discrepancies between these three models suggest the ion distribution, boundary conditions, and symmetries of the system may have a significant impact on the absorption in warm dense matter at low temperatures.

This observation of significant changes to the free-free opacity induced by melting is consistent with the recent measurements of Williams et al. [14], who also attributed their observation to the breakdown of the crystal structure. However, here we predict larger opacity increases than those reported by Williams et al., by about a factor of 2 . We note that our calculated opacities are also larger than those previously published by Vinko et al. [9] for both cold and warm systems.

For $\hbar \omega<25 \mathrm{eV}$ the temperature dependence of the opacity is consistent with plasmon broadening due to increased electron-ion collisions [31]. For $35<\hbar \omega<60 \mathrm{eV}$ only a relatively weak temperature dependence is predicted with the exception of the $2 s-2 p$ resonance ( $\hbar \omega \approx 42 \mathrm{eV}$ ) owing to thermal ionization of the L-shell at the highest temperatures. We identify this weak dependence as partly a consequence of LFC effects enhancing the opacity at $T=1 \mathrm{eV}$. The strong impact of LFC at $T=1 \mathrm{eV}$ further suggests the importance of ion-ion correlations at this temperature. Close to the L-edge, significant pre-edge features are predicted to develop as low lying continuum states are thermally depopulated. This should be distinguished from changes in the continuum lowering 


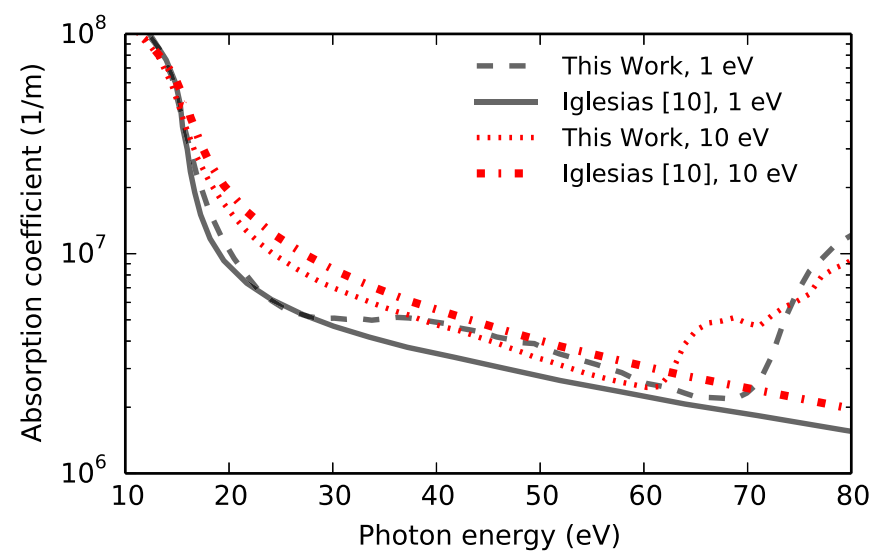

FIG. 8. Comparison of our calculated opacity with the inverse bremsstrahlung (IB) model of Ref. [10] at high temperatures. Our results appear to approach the IB model both in slope and absolute value.

which remains relatively constant, only changing by $\approx 4 \mathrm{eV}$ for the highest temperature of $15 \mathrm{eV}$.

Large scale DFT, and in particular $\mathrm{G}_{0} \mathrm{~W}_{0}$, calculations are expensive at higher temperatures. As such, there is a clear incentive to establish under what conditions simpler models, such as IB calculations, may be sufficiently accurate. In Fig. 8 we plot our finite-temperature calculations for 1 and $10 \mathrm{eV}$ along with the IB model of Ref. [10]. The authors of Ref. [10] used a frozen core pseudopotential and therefore cannot be expected to replicate the L-edge features or effects due to L-shell ionization such as the appearance of the $2 s-2 p$ bound-bound transition. Nonetheless, the IB calculations closely agree with our results for $\hbar \omega<25 \mathrm{eV}$ at a temperature of $1 \mathrm{eV}$ where the opacity is dominated by the plasmon feature. At higher temperatures, but still sufficiently cool that thermal ionisation of the L-shell remains low, our calculations do appear to be approaching the IB opacity model both in slope and absolute values.

In conclusion, we present measurements and calculations of the free-free opacity in Al between the plasma frequency and the L-shell absorption edge. Our experimental data shows good agreement with previous measurements by Henke et al. [24] and Birken et al. [26], and disagrees with the measurements by Kettle et al. [12], and with Gullikson et al. [23] at lower photon energies. We find excellent agreement between our measurements and full time-dependent DFT calculations of the free-free opacity. We extend our simulations up to temperatures of $15 \mathrm{eV}$, demonstrating and quantifying the influence of finite-temperature $\mathrm{G}_{0} \mathrm{~W}_{0}$ corrections in warm dense matter conditions. IB-based plasma models show good agreement with our calculations for temperatures above around $10 \mathrm{eV}$. This result is particularly encouraging given the substantial difficulties in conducting full GW-DFT-MD simulations at higher temperatures and provides confidence in more approximate but faster plasma models for radiative properties of warm dense matter. Finally, our results demonstrate the importance of finite-temperature quasiparticle and LFC in DFT-based modeling, with important implications for the evaluation of the $a b$ initio dielectric response more generally, including calculations of dynamic structure factors and stopping powers.

\section{ACKNOWLEDGMENTS}

We wish to thank the Target Fabrication Group at the Central Laser Facility for manufacturing and characterizing our target samples. P.H., J.S.W., and S.M.V. acknowledge support from the U.K. EPSRC under Grant No. EP/P015794/1. S.M.V. gratefully acknowledges support from the Royal Society.
[1] H. A. Kramers, Philos. Mag. 46, 836 (1923).

[2] R. R. Johnston, J. Quant. Spectrosc. Radiat. Transfer 7, 815 (1967).

[3] J. F. Seely and E. G. Harris, Phys. Rev. A 7, 1064 (1973).

[4] V. N. Tsytovich, R. Bingham, U. de Angelis, and A. Forlani, Phys. Lett. A 205, 199 (1995).

[5] H. Totsuji, Phys. Rev. A 32, 3005 (1985).

[6] J. Dawson and C. Oberman, Phys. Fluids 5, 517 (1962).

[7] G. Bekefi, Radiation Processes in Plasmas (Wiley, New York, 1966).

[8] A. Ron and N. Tzoar, Phys. Rev. 131, 12 (1963).

[9] S. M. Vinko, G. Gregori, M. P. Desjarlais, B. Nagler, T. J. Whitcher, R. W. Lee, P. Audebert, and J. S. Wark, High Energy Density Phys. 5, 124 (2009).

[10] C. A. Iglesias, High Energy Density Phys. 6, 311 (2010).

[11] G. O. Williams, H.-K. Chung, S. M. Vinko, S. Künzel, A. B. Sardinha, P. Zeitoun, and M. Fajardo, Phys. Plasmas 20, 042701 (2013).

[12] B. Kettle, T. Dzelzainis, S. White, L. Li, B. Dromey, M. Zepf, C. L. S. Lewis, G. Williams, S. Kunzel, M. Fajardo, H. Dacasa,
P. Zeitoun, A. Rigby, G. Gregori, C. Spindloe, R. Heathcote, and D. Riley, Phys. Rev. E 94, 023203 (2016).

[13] N. R. Shaffer, N. G. Ferris, J. Colgan, D. P. Kilcrease, and C. E. Starrett, High Energy Density Phys. 23, 31 (2017).

[14] G. O. Williams, S. Kunzel, S. Daboussi, B. Iwan, A. I. Gonzalez, W. Boutu, V. Hilbert, U. Zastrau, H. J. Lee, B. Nagler, E. Granados, E. Galtier, P. Heimann, B. Barbrel, G. Dovillaire, R. W. Lee, J. Dunn, V. Recoules, C. Blancard, P. Renaudin, A. G. de la Varga, P. Velarde, P. Audebert, H. Merdji, P. Zeitoun, and M. Fajardo, Phys. Rev. A 97, 023414 (2018).

[15] J. J. Hopfield, Phys. Rev. 139, A419 (1965).

[16] K. Sturm and E. Pajanne, J. Phys. F 3, 199 (1973).

[17] K. Sturm, Adv. Phys. 31, 1 (1982).

[18] K. Sturm, E. Zaremba, and K. Nuroh, Phys. Rev. B 42, 6973 (1990).

[19] A. B. Zylstra, J. A. Frenje, P. E. Grabowski, C. K. Li, G. W. Collins, P. Fitzsimmons, S. Glenzer, F. Graziani, S. B. Hansen, S. X. Hu, M. G. Johnson, P. Keiter, H. Reynolds, J. R. Rygg, F. H. Seguin, and R. D. Petrasso, Phys. Rev. Lett. 114, 215002 (2015). 
[20] D. Roth, B. Bruckner, M. V. Moro, S. Gruber, D. Goebl, J. I. Juaristi, M. Alducin, R. Steinberger, J. Duchoslav, D. Primetzhofer, and P. Bauer, Phys. Rev. Lett. 118, 103401 (2017).

[21] A. D. Baczewski, L. Shulenburger, M. P. Desjarlais, S. B. Hansen, and R. J. Magyar, Phys. Rev. Lett. 116, 115004 (2016).

[22] CXRO Database, http://henke.lbl.gov/optical_constants/.

[23] E. M. Gullikson, P. Denham, S. Mrowka, and J. H. Underwood, Phys. Rev. B 49, 16283 (1994).

[24] B. L. Henke, E. M. Gullikson, and J. C. Davis, At. Data Nucl. Data Tables 54, 181 (1993).

[25] R. Keenan, C. L. S. Lewis, J. S. Wark, and E. Wolfrum, J. Phys. B 35, L447 (2002).

[26] H. G. Birken, W. Jark, C. Kunz, and R. Wolf, Nucl. Instrum. Methods 253, 166 (1986).

[27] F. Frassetto, C. Cacho, C. A. Froud, I. E. Turcu, P. Villoresi, W. A. Bryan, E. Springate, and L. Poletto, Opt. Express 19, 19169 (2011).

[28] P. E. Blöchl, Phys. Rev. B 50, 17953 (1994).

[29] M. Torrent, F. Jollet, F. Bottin, G. Zérah, and X. Gonze, Comput. Mater. Sci. 42, 337 (2008).

[30] C. E. Starrett and D. Saumon, Phys. Rev. E 93, 063206 (2016).

[31] B. B. L. Witte, L. B. Fletcher, E. Galtier, E. Gamboa, H. J. Lee, U. Zastrau, R. Redmer, S. H. Glenzer, and P. Sperling, Phys. Rev. Lett. 118, 225001 (2017).

[32] N. T. Maitra, J. Chem. Phys. 144, 220901 (2016).

[33] R. van Leeuwen, Phys. Rev. Lett. 82, 3863 (1999).

[34] N. T. Maitra and K. Burke, Phys. Rev. A 63, 042501 (2001).

[35] M. W. C. Dharma-wardana, Computation 4, 16 (2016).

[36] F. Perrot and M. W. C. Dharma-wardana, Phys. Rev. A 30, 2619 (1984).

[37] M. W. C. Dharma-wardana and R. Taylor, J. Phys. C 14, 629 (1981).

[38] F. Perrot and M. W. C. Dharma-wardana, Phys. Rev. B 62, 16536 (2000).

[39] A. Pribram-Jones, P. E. Grabowski, and K. Burke, Phys. Rev. Lett. 116, 233001 (2016).
[40] Abinit Website, http://www.abinit.org.

[41] X. Gonze et al., Comput. Phys. Commun. 205, 106 (2016).

[42] T. Miyake and F. Aryasetiawan, Phys. Rev. B 61, 7172 (2000).

[43] M. Shishkin and G. Kresse, Phys. Rev. B 74, 035101 (2006).

[44] G. Onida, L. Reining, and A. Rubio, Rev. Mod. Phys. 74, 601 (2002).

[45] L. Hedin, Phys. Rev. 139, A796 (1965).

[46] L. Hedin, J. Phys.: Condens. Matter 11, R489 (1999).

[47] S. V. Faleev, M. van Schilfgaarde, T. Kotani, F. Léonard, and M. P. Desjarlais, Phys. Rev. B 74, 033101 (2006).

[48] R. Zimmermann, K. Kilimann, W. D. Kraeft, D. Kremp, and G. Röpke, Phys. Status Solidi B 90, 175 (1978).

[49] G. Röpke, K. Kilimann, D. Kremp, W. D. Kraeft, and R. Zimmermann, Phys. Status Solidi B 88, K59 (1978).

[50] W.-D. Kraeft, D. Kremp, W. Ebeling, and G. Röpke, Quantum Statistics of Charged Particle Systems (Akademie-Verlag, Berlin, and Plenum Press, London/New York, 1986).

[51] S. Günter, L. Hitzschke, and G. Röpke, Phys. Rev. A 44, 6834 (1991).

[52] J. Seidel, S. Arndt, and W. D. Kraeft, Phys. Rev. E 52, 5387 (1995).

[53] D. Kremp, M. Schlanges, W.-D. Kraeft, and T. Bornath, Quantum Statistics of Nonideal Plasmas (Springer-Verlag, Berlin, Heidelberg, 2005).

[54] C. Lin, G. Röpke, W. D. Kraeft, and H. Reinholz, Phys. Rev. E 96, 013202 (2017).

[55] J. J. Kas and J. J. Rehr, Phys. Rev. Lett. 119, 176403 (2017).

[56] S. Lebègue, B. Arnaud, M. Alouani, and P. E. Bloechl, Phys. Rev. B 67, 155208 (2003).

[57] H. S. Yamada and K. S. Ikeda, Intl. J. Computat. Math. 2014, 587430 (2014).

[58] A. Östlin, L. Vitos, and L. Chioncel, Phys. Rev. B 96, 125156 (2017).

[59] Y. Pavlyukh, Sci. Rep. 7, 504 (2017).

Correction: An alternate email address was missing at publication and has now been inserted. 